\title{
Calcium Intake and Serum Calcium Level in Relation to the Risk of Ischemic Stroke: Findings from the REGARDS Study
}

\author{
Daniel T. Dibaba, ${ }^{\mathrm{a}, \mathrm{b}}$ Pengcheng Xun, ${ }^{\mathrm{a}}$ Alyce D. Fly, ${ }^{\mathrm{a}}$ Aurelian Bidulescu, ${ }^{\mathrm{a}}$ Cari L. Tsinovoi, ${ }^{\mathrm{a}}$ \\ Suzanne E. Judd, ${ }^{\mathrm{c}}$ Leslie A. McClure, ${ }^{\mathrm{d}}$ Mary Cushman, ${ }^{\mathrm{e}}$ Frederick W. Unverzagt, ${ }^{\mathrm{f}} \mathrm{Ka} \mathrm{He}^{\mathrm{a}, \mathrm{g}}$ \\ ${ }^{a}$ Department of Epidemiology and Biostatistics, School of Public Health, Indiana University Bloomington, Bloomington, IN, USA \\ ${ }^{b}$ Department of Vice Chancellor for Research/Tennessee Clinical and Translational Science Institute, University of Tennessee Health Science \\ Center, Memphis, TN, USA \\ 'Department of Biostatistics, School of Public Health, University of Alabama at Birmingham, Birmingham, AL, USA \\ ${ }^{d}$ Department of Epidemiology and Biostatistics, Dornsife School of Public Health, Drexel University, Philadelphia, PA, USA \\ eDepartment of Medicine, Larner College of Medicine, University of Vermont, Burlington, VT, USA \\ fDepartment of Psychiatry, School of Medicine, Indiana University, Indianapolis, IN, USA \\ ${ }^{9}$ Department of Obstetrics and Gynecology, Columbia University Irving Medical Center, New York, NY, USA
}

Background and Purpose Data on the association between calcium ( $\mathrm{Ca}$ ) and ischemic stroke are sparse and inconsistent. This study aimed to examine $\mathrm{Ca}$ intake and serum $\mathrm{Ca}$ levels in relation to risk of ischemic stroke.

Methods The primary analysis included 19,553 participants from the Reasons for Geographic And Racial Differences in Stroke (REGARDS) study. A subcohort was randomly selected to create a casecohort study $(n=3,016)$, in which serum Ca levels were measured. Ischemic stroke cases were centrally adjudicated by physicians based on medical records. Cox proportional hazards regression for the cohort and weighted Cox proportional hazard regression with robust sandwich estimation method for the case-cohort analysis with adjustment for potential confounders were performed.

Results During a mean 8.3-year follow-up, 808 incident cases of ischemic stroke were documented. Comparing the highest quintile to the lowest, a statistically significant inverse association was observed between total $\mathrm{Ca}$ intake and risk of ischemic stroke (hazard ratio [HR], 0.72; 95\% confidence interval $[\mathrm{Cl}], 0.55$ to $\left.0.95 ; P_{\text {inear-trend }}=0.183\right)$; a restricted cubic spline analysis indicated a threshold effect like non-linear association of total $\mathrm{Ca}$ intake with ischemic stroke $\left(P_{\text {non-linear }}=0.006\right)$. In the case-cohort, serum Ca was inversely associated with the risk of ischemic stroke. Compared to the lowest, the highest quintile of serum $\mathrm{Ca}$ had a $27 \%$ lower risk of ischemic stroke ( $\mathrm{HR}, 0.73$; $95 \% \mathrm{Cl}, 0.53$ to $\left.0.99 ; P_{\text {linear-trend }}=0.013\right)$. Observed associations were mainly mediated by type 2 diabetes, hypertension, and cholesterol.

Conclusions These findings suggest that serum Ca has inverse and Ca intake has threshold effect like association with risk of ischemic stroke.
Correspondence: Ka He Department of Obstetrics and Gynecology, Columbia University Irving Medical Center, 622 W. 168th, Room 16-62, New York, NY 10032, USA Tel: +1-212-305-6973

Fax:+1-212-305-2171 E-mail: kk3399@cumc.columbia.edu

Received: March 8, 2019

Revised: June 28, 2019

Accepted: August 27, 2019

Keywords Stroke; Calcium; Hypertension; Diabetes mellitus; Cholesterol

Copyright ( ) 2019 Korean Stroke Society

This is an Open Access article distributed under the terms of the Creative Commons Attribution Non-Commercial License (http://creativecommons.org/licenses/by-nc/4.0/) which permits unrestricted non-commercial use, distribution, and reproduction in any medium, provided the original work is properly cited. 


\section{Introduction}

Because of its favorable effects on blood pressure, ${ }^{1,2}$ hypertension, ${ }_{1}^{3,4}$ type 2 diabetes, ${ }_{1}^{5}$ insulin resistance, ${ }_{1}^{6}$ and metabolic syndrome, ${ }^{7}$ calcium (Ca) may play an important role in the prevention of ischemic stroke. Results from meta-analyses of studies exploring the association between dietary $\mathrm{Ca}$ intake and the risk of ischemic stroke are inconsistent but suggest a non-linear trend. ${ }^{8,9}$ In 2013, a dose-response meta-analysis demonstrated an inverse association between dietary $\mathrm{Ca}$ intake and ischemic stroke risk in participants with low $\mathrm{Ca}$ intake (i.e., $<700 \mathrm{mg} /$ day), but a positive borderline association between $\mathrm{Ca}$ intake and the risk of ischemic stroke in participants with $>700 \mathrm{mg} /$ day. ${ }^{8}$ A more recent meta-analysis of 10 cohort studies found no association between dietary $\mathrm{Ca}$ intake and total stroke or stroke subtypes but predicted a non-linear association between dietary $\mathrm{Ca}$ intake and the risk of total stroke. ${ }^{9}$ However, the study found those with high Ca intake from dairy sources and those with longer follow-up period ( $\geq 14$ years) had reduced risk of total stroke.

Although bioavailability of $\mathrm{Ca}$ intake is always a concern, studies on the association between serum $\mathrm{Ca}$ and the risk of ischemic stroke are sparse. In a prospective study, serum Ca was associated with increased risk of ischemic stroke. ${ }^{10}$ Moreover, a meta-analysis of three cohort studies reported a direct association between circulating Ca levels and risk of total stroke."

There is a growing concern about the role of $\mathrm{Ca}$ intake at high levels in elevating the risk of heart diseases and stroke. ${ }^{12-15}$ Nonetheless, no prospective cohort study has examined both $\mathrm{Ca}$ intake and serum Ca levels in relation to the risk of ischemic stroke in the same study. In the present study, we aimed to investigate the association of $\mathrm{Ca}$ intake and serum $\mathrm{Ca}$ level with the risk of ischemic stroke. In addition, we aimed to investigate whether any observed association was modified by sex, race, and region using data from the Reasons for Geographic And Racial Differences in Stroke (REGARDS) study.

\section{Methods}

The REGARDS study is a longitudinal population-based study among blacks and whites designed to investigate risk factors associated with excess stroke mortality in the USA among blacks and residents of the Stroke Belt region. ${ }^{16}$ The prospective participants in the REGARDS study were randomly selected from commercially available nationwide lists purchased through GeneSys, Inc. (Daly City, CA, USA). ${ }^{17}$ Prospective participants were sent letters by mail to introduce the study, and then they were contacted by telephone for recruitment between January 2003 and 0c- tober 2007. Demographic data were collected using computerassisted telephone interviews after initial verbal consent. During the subsequent baseline home visits, written informed consents were obtained and dietary and behavioral data were collected by self-administered questionnaires. Physical measurements including weight, height, and cardiovascular health profile (electrocardiography and blood pressures) were recorded and blood samples were collected during baseline first home visits. The REGARDS cohort has been described in more detail elsewhere. ${ }^{16,18}$ Of the initially recruited 30,239 participants, 10,509 participants were excluded due to missing data on dietary intake, income, stroke history, or other covariates. Additionally, we excluded 177 participants ( $n=90$ incident hemorrhagic stroke cases, $n=87$ unidentified stroke cases) leaving 19,553 participants for the cohort analysis (Figure 1). None of the participants had an implausible value for total energy intake ( $<500 \mathrm{kcal} /$ day or $>5,000 \mathrm{kcal} /$ day ${ }^{19}$ ) in the data included in this study.

We also conducted a case-cohort analysis investigating the association of serum Ca levels with the risk of ischemic stroke. The subcohort was randomly selected from the REGARDS cohort using stratified random sampling from each stratum jointly classified by age, sex, race, and region with an overall sampling probability of $9 \%$, and all incident stroke cases were included $(n=730)$. In the case-cohort analysis, a total of 3,016 participants (with 730 incident ischemic stroke cases, 86 of which were in the random subcohort) that had data for serum Ca were included after excluding those with non-ischemic stroke cases ( $n=55$ hemorrhagic stroke), and those with missing values in key covariates ( $n=293$ ) (Figure 2). The final weighted effective sample size for the case-cohort was 3,102. The REGARDS study

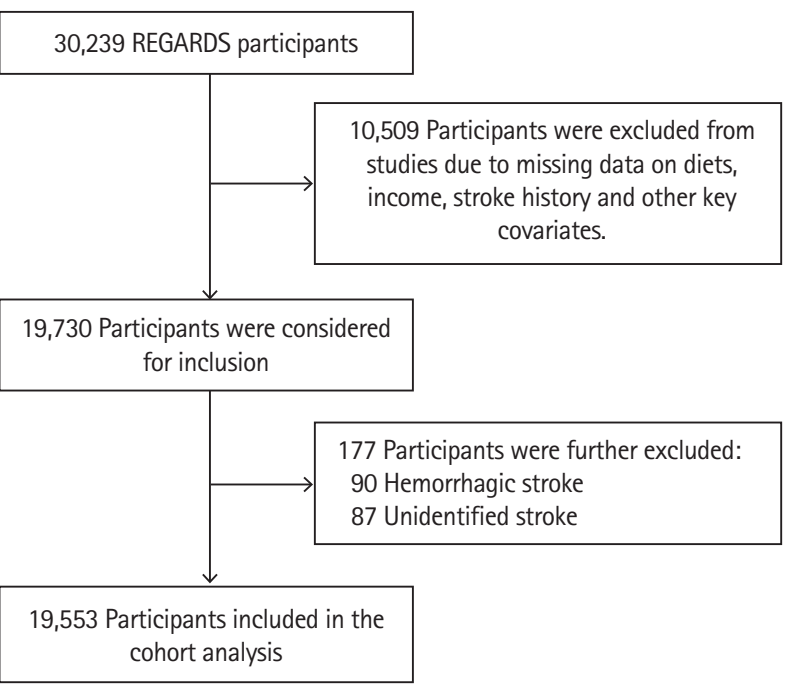

Figure 1. Study participants' selection process in cohort analysis. REGARDS, Reasons for Geographic And Racial Differences in Stroke. 


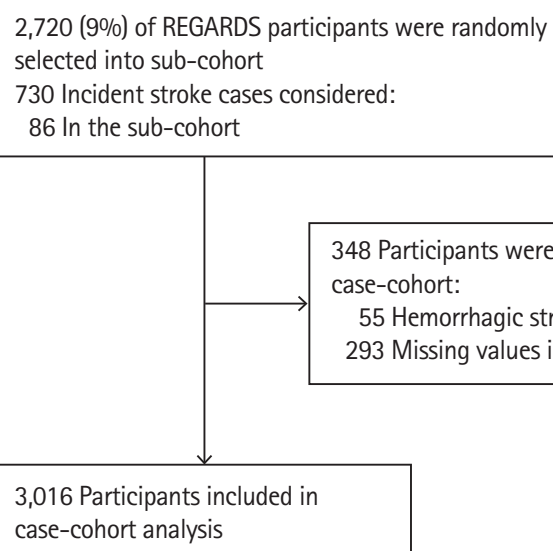

3,016 Participants included in case-cohort analysis

Figure 2. Study participants' selection process in case-cohort analysis. REGARDS, Reasons for Geographic And Racial Differences in Stroke.

was approved by Institutional Review Boards (IRB) of each participating institution, and the present study was approved by the IRB for Indiana University, Bloomington.

\section{Exposure measurement}

Dietary intake data were collected using a self-administered modified Block 98 food frequency questionnaire (FFO) that had over 150 multiple choice questions about 107 food items. ${ }^{18,20,21}$ The FFO asked the frequency and amount of intake of each food item per day over the past 1 year at baseline. Nutrients in foods were extracted by NutritionQuest (Berkeley, CA, USA) using the Block nutrient database that was developed based on the United States Department of Agriculture Nutrient Database for Standard References. ${ }^{22}$ The amount of each food consumed was calculated by multiplying the reported frequency by the portion size for each food item. The total amount of a nutrient contributed from each food was derived by multiplying the amount consumed by the amount of the nutrient in the given FFO line item. Nutrients were summed over all FFO food items to provide estimates for total daily nutrient intake. The Block FFO had been validated for the assessment of nutrients including $\mathrm{Ca}$ with a correlation coefficient greater than 0.6 for $\mathrm{Ca}$ between Block FFO and multiple-day 24-hour recall. ${ }^{23,24}$ Total (dietary plus supplementation) and dietary (from food only) $\mathrm{Ca}$ intake were reported as milligram per day (mg/day). Supplemental $\mathrm{Ca}$ intake was assessed through identification in a medication inventory whereby the participants showed a trained health professional any medication they used at least once in the 2 weeks prior to the first in-home visit, and then Ca was estimated from actual Ca supplements, multivitamins or Ca-containing medications.

A blood sample was collected using standard phlebotomy during the first home visit at baseline. Among participants included in the case-cohort study, serum Ca was measured using an automated enzyme colorimetric assay on the Roche Integra instrument (Roche Diagnostics, Indianapolis, IN, USA). The assay range was 0.4 to $100 \mathrm{mg} / \mathrm{dL}$. Inter-assay coefficients of variation ranged from $1.88 \%$ to $2.82 \%$.

\section{Outcome ascertainment}

The REGARDS study participants were followed every 6 months via telephone for stroke events and to obtain the reason for hospitalization if a participant was hospitalized. Medical records were obtained and evaluated if the participant reported seeking medical care for stroke events, transient ischemic attack, or death. ${ }^{25}$ Once the medical record was received for a stroke event, a team of physicians verified and subtyped the stroke event as ischemic or hemorrhagic. ${ }^{25,26}$ Ischemic stroke was defined according to the World Health Organization ${ }^{27}$ as sudden focal, at times global neurological deficits lasting $>24$ hours with signs and symptoms corresponding to the involvement of focal areas of the brain or as a non-focal neurological deficit with imaging consistent with that of ischemic stroke. ${ }^{16,28}$

\section{Covariates}

Data collected using computer-assisted telephone interview included age (continuous), sex (female/male), race (black/white), regions, body mass index (BMI), socioeconomic status (education and income), regular aspirin use (yes or no), smoking, alcohol intake, food intake, and physical activity level. Region was categorized into non-Belt, Stroke Belt (Alabama, Arkansas, Georgia, Louisiana, Mississippi, North Carolina, South Carolina, and Tennessee), or Stroke-Buckle (coastal plains in Georgia, North Carolina, and South Carolina with high stroke mortality). ${ }^{29}$ Sex was self-reported. Education was categorized to less than high school, high school, some college, and college plus. Annual income was categorized to $<20,20$ to 34,35 to 74 , and $\geq 75$ USD. BMI was derived from weight in $\mathrm{kg}$ divided by height in meters squared. Alcohol use was categorized into three levels based on drinks per week, either none, moderate ( 0 to 7 drinks) week in women, 0 to 14 drinks/week in men), or heavy ( $>7$ drinks/week in women, $>14$ drinks/week in men). Smoking was categorized as never smoker, former smoker, or current smoker. Exercise was categorized into three levels based on the frequency of physical activity per week (none, 1 to 3 times, or 4 or more times). Two blood pressure measurements were taken using aneroid sphygmomanometer during home visits and the mean of the two measurements was recorded. Total calorie intake and vitamin $D$ intake were generated from the FFO. Other covariates including total cholesterol, high density lipoprotein 
(HDL), and low density lipoprotein (LDL), and blood glucose levels were measured from the blood sample taken during the baseline in-home visit by colorimetric reflectance spectrophotometry using the Ortho Vitros Clinical Chemistry System 950IRC instrument (Johnson \& Johnson Clinical Diagnostics, New Brunswick, NJ, USA). ${ }^{18}$ Serum 25-hydroxyvitamin D $[25(\mathrm{OH}) \mathrm{D}]$ in $\mathrm{ng} / \mathrm{mL}$ ) was generated from an ancillary study to adjust for in sensitivity analysis in the case-cohort analysis. Because $\mathrm{Ca}$ and magnesium (Mg) compete for intestinal absorption and renal reabsorption, we also adjusted for $\mathrm{Mg}$ intake in the cohort and serum $\mathrm{Mg}$ in the case-cohort models. ${ }^{30,31}$

\section{Statistical analysis}

Analysis of variance (ANOVA; for normally distributed continuous variables), and Kruskal-Wallis test (for non-normally distributed continuous variables and for ordinal variables) were used to compare covariates across Ca quintiles. We used the chi-square test to compare the distribution of categorical variables across $\mathrm{Ca}$ quintiles.

We compared the risk of ischemic stroke by the levels of total, dietary, and supplemental $\mathrm{Ca}$ intake using Cox proportional hazards regression models adjusted for covariates in a sequential manner: model 1 included the exposure of interest, age sex, race, BMI, region and the interaction of age and race. Model 2 additionally adjusted for education, income, smoking, alcohol consumption, and exercise. Model 3 further adjusted for total energy intake, regular aspirin use, and total $\mathrm{Mg}$ intake. The model on dietary $\mathrm{Ca}$ intake was adjusted for supplemental $\mathrm{Ca}$ intake, and the model on supplemental $\mathrm{Ca}$ intake was adjusted for dietary Ca intake, respectively. Model 4 was further adjusted for HDL, and vitamin D intake. Ca was categorized in quintiles in the analyses. To assess trend across quintiles of $\mathrm{Ca}_{\text {, }}$ the median of each quintile, as a continuous variable, was included in the models. We also examined non-linear associations between $\mathrm{Ca}$ and the risk of ischemic stroke non-parametrically with restricted cubic spline analyses. ${ }^{32}$ In the cubic spline analysis, we used the median of the first quintile of $\mathrm{Ca}$ as the reference and 4 knots. For supplemental Ca intake we used 50 $\mathrm{mg} /$ day as the reference category; participants in the first quintile of supplemental Ca did not consume a Ca supplement.

We further conducted a stratified analysis to determine whether there was a difference in the associations by sex, race, and region. Interactions between variables in the models were considered significant at $P$-values $\leq 0.1$. We examined whether hypertension, systolic blood pressure (SBP), fasting blood glucose (FBG), type 2 diabetes, total cholesterol, HDL, LDL, or triglyceride mediated the potential association between $\mathrm{Ca}$ and ischemic stroke each at a time using the method described by
Hertzmark et al. (2012). ${ }^{33}$ This method calculates the proportion mediated as percent mediated $=\left[1-\left(\frac{\beta_{2}}{\beta_{1}}\right)\right] \times 100 \%$, where $\beta_{1}$ and $\beta_{2}$ are regression coefficients from the model with and without intermediator, respectively. The standard error was calculated based on the covariance matrix between the two models. The covariates in the final model of the main analysis were included in the mediation analysis and are assumed to meet the confounding assumptions of mediation analysis. ${ }^{34}$ We used the median of each quintile of $\mathrm{Ca}$ intake as the exposure and categorized the intermediate variables as a binary variable. We categorized total cholesterol $\geq 200 \mathrm{mg} / \mathrm{dL}$ as high level. We also used continuous forms of total cholesterol, HDL, LDL, SBP, and FBG and binary forms for type 2 diabetes and hypertension for mediation analysis.

For the case-cohort analysis, we used a weighted multivariable Cox proportional hazards regression to compute the parameters and robust variance estimates. We weighted noncase subcohort by the inverse of subcohort sampling fraction. Case subcohort was assigned a weight equal to the inverse of subcohort sampling fraction up to shortly before the failure time and a weight equal to 1 from that point to failure time. The non-subcohort cases were assigned a weight of 1 and entered the risk set shortly before failure time. ${ }^{35}$ This analysis was based on the method provided by Barlow et al. ${ }^{36}$ that has been explained in detail by Kulathinal et al. ${ }^{35}$

The proportional hazard regression assumptions were tested by plotting the cumulative martingale residuals against the variable of interest and using the Supremum test. We considered $P$ values $\leq 0.05$ statistically significant if not otherwise specified. We conducted all the statistical analyses in SAS version 9.4 (SAS Institute Inc., Cary, NC, USA).

\section{Data availability}

Any reasonable request for the REGARDS datasets used in this study should be submitted to the REGARDS Publication and Presentation Subcommittee via http://www.regardsstudy.org and the datasets are available upon approval.

\section{Results}

During a mean follow-up of 8.3-year (standard deviation: 3.2), 808 incident cases of ischemic stroke were observed in the cohort. At baseline, participants with higher total $\mathrm{Ca}$ intake were more likely to be older, non-smokers, more likely to have lower BMI. Participants with high total Ca had lower diastolic blood pressure and SBP, were less likely to have type 2 diabetes, were more likely to consume alcohol at moderate levels, exercise 4 or more times per week, and have used $\mathrm{Ca}$ and $\mathrm{Mg}$ supple- 
Table 1. Baseline characteristics of participants in the cohort by quintiles of total Ca intakes: the REGARDS study $(n=19,553)$

\begin{tabular}{|c|c|c|c|c|c|c|}
\hline \multirow{2}{*}{ Characteristic } & \multicolumn{5}{|c|}{ Ca intake } & \multirow{2}{*}{$P$} \\
\hline & Quintile 1 & Quintile 2 & Quintile 3 & Quintile 4 & Quintile 5 & \\
\hline Total Ca $(\mathrm{mg} / \mathrm{day})^{*}$ & $344.6 \pm 90.1$ & $588.28 \pm 63.9$ & $854.3 \pm 97.6$ & $1,309.1 \pm 151.6$ & $1,925.4 \pm 319.8$ & \\
\hline Dietary Ca (mg/day) & $317.1 \pm 90.3$ & $521.38 \pm 105.1$ & $748.2 \pm 156.1$ & $759.2 \pm 399.5$ & $957.9 \pm 421.7$ & $<0.0001$ \\
\hline Supplementary Ca (mg/day) & $27.4 \pm 52.3$ & $66.90 \pm 88.3$ & $106.1 \pm 134.0$ & $549.9 \pm 459.0$ & $967.5 \pm 316.8$ & $<0.0001$ \\
\hline Total Mg (mg/day)* & $190.9 \pm 69.9$ & $284.94 \pm 86.8$ & $351.3 \pm 103.0$ & $348.4 \pm 136.2$ & $422.3 \pm 133.8$ & $<0.0001$ \\
\hline Dietary Mg (mg/day) & $175.7 \pm 62.0$ & $247.15 \pm 80.4$ & $305.6 \pm 97.0$ & $297.6 \pm 130.1$ & $350.1 \pm 131.2$ & $<0.0001$ \\
\hline Supplementary Mg (mg/day) & $20.9 \pm 37.8$ & $37.79 \pm 45.5$ & $45.8 \pm 46.4$ & $50.7 \pm 46.8$ & $72.2 \pm 42.8$ & $<0.0001$ \\
\hline Age (yr) & $63.8 \pm 9.0$ & $64.34 \pm 9.2$ & $64.6 \pm 9.4$ & $65.0 \pm 9.1$ & $66.4 \pm 9.2$ & $<0.0001$ \\
\hline Female sex (\%) & 55.12 & 46.7 & 43.62 & 61.3 & 71.02 & $<0.0001$ \\
\hline Black race (\%) & 49.10 & 37.7 & 30.58 & 27.6 & 18.67 & $<0.0001$ \\
\hline BMI $\left(\mathrm{kg} / \mathrm{m}^{2}\right)$ & $29.8 \pm 6.2$ & $29.5 \pm 6.0$ & $29.1 \pm 5.9$ & $28.8 \pm 6.0$ & $28.0 \pm 5.9$ & $<0.0001$ \\
\hline Alcohol (\%) & & & & & & $<0.0001$ \\
\hline Heavy & 4.9 & 4.7 & 4.1 & 4.1 & 4.0 & \\
\hline Moderate & 31.1 & 37.7 & 38.5 & 36.7 & 36.9 & \\
\hline None & 64.0 & 57.6 & 57.4 & 59.2 & 59.1 & \\
\hline Type 2 diabetes (\%) & 21.6 & 20.5 & 20.2 & 15.6 & 13.0 & $<0.0001$ \\
\hline Diastolic BP (mm Hg) & $77.1 \pm 9.8$ & $76.59 \pm 9.4$ & $76.4 \pm 9.5$ & $75.7 \pm 9.6$ & $74.8 \pm 9.0$ & $<0.0001$ \\
\hline Systolic BP (mm Hg) & $128.4 \pm 16.8$ & $127.33 \pm 16.2$ & $126.9 \pm 16.5$ & $125.9 \pm 16.5$ & $124.9 \pm 15.7$ & $<0.0001$ \\
\hline Hypertension (\%) & 62.3 & 58.3 & 55.3 & 54.3 & 53.0 & $<0.0001$ \\
\hline Smoking (\%) & & & & & & $<0.0001$ \\
\hline Current & 18.9 & 15.5 & 12.9 & 12.9 & 8.9 & \\
\hline Past & 39.1 & 43.9 & 43.3 & 40.9 & 38.9 & \\
\hline Never & 42.0 & 40.7 & 43.8 & 47.8 & 52.2 & \\
\hline \multicolumn{7}{|l|}{ Exercise (time/wk) } \\
\hline$\geq 4$ & 26.2 & 28.8 & 32.0 & 31.0 & 34.5 & $<0.0001$ \\
\hline $1-3$ & 35.8 & 37.9 & 37.6 & 37.5 & 36.6 & \\
\hline None & 38.0 & 33.3 & 30.4 & 31.4 & 28.9 & \\
\hline Aspirin use (\%) & 40.0 & 43.3 & 45.0 & 45.7 & 47.4 & $<0.0001$ \\
\hline Total cholesterol (mg/dL) & $192.3 \pm 41.1$ & $190.3 \pm 39.9$ & $190.0 \pm 39.5$ & $194.1 \pm 39.0$ & $193.3 \pm 37.9$ & $<0.0001$ \\
\hline HDL (mg/dL) & $51.1 \pm 15.7$ & $50.5 \pm 15.9$ & $50.0 \pm 15.6$ & $53.5 \pm 16.7$ & $55.7 \pm 16.7$ & $<0.0001$ \\
\hline $\mathrm{LDL}(\mathrm{mg} / \mathrm{dL})$ & $115.6 \pm 35.8$ & $113.3 \pm 34.7$ & $113.2 \pm 34.2$ & $113.9 \pm 34.1$ & $111.8 \pm 32.9$ & 0.0002 \\
\hline Triglyceride (mg/dL) & $129.1 \pm 78.2$ & $134.1 \pm 85.8$ & $136.4 \pm 85.4$ & $135.6 \pm 90.4$ & $130.7 \pm 85.5$ & $<0.0001$ \\
\hline Calorie intake (kcal/day) & $1,216.7 \pm 402$ & $1,627.7 \pm 541.9$ & $1,931.4 \pm 667$ & $1,799.9 \pm 828.1$ & $1,965.8 \pm 741$ & $<0.0001$ \\
\hline Vitamin D (IU/day) & $70.4 \pm 46.0$ & $113.2 \pm 65.0$ & $160.4 \pm 92.0$ & $166.7 \pm 135.6$ & $204.7 \pm 154.0$ & $<0.0001$ \\
\hline
\end{tabular}

Values are presented as mean \pm standard deviation. Analysis of variance and Kruskal-Wallis test were used to compare continuous variables across nutrient quintiles. Chi-square test was used to test the association of categorical variables with quintiles of $\mathrm{Ca}$.

REGARDS, Reasons for Geographic And Racial Differences in Stroke; Ca, calcium; Mg, magnesium; BMI, body mass index; BP, blood pressure; HDL, high density lipoprotein; LDL, low density lipoprotein; IU, international unit.

*Total intake includes both dietary and supplemental intake.

ments and aspirin regularly $(P<0.05)$ (Table 1$)$.

When comparing the highest quintile to the lowest quintile of total $\mathrm{Ca}$ intake, total $\mathrm{Ca}$ was significantly inversely associated with ischemic stroke (hazard ratio [HR], 0.72; 95\% confidence interval $[\mathrm{Cl}], 0.55$ to $0.95 ; P_{\text {linear-trend }}=0.183$ ) (Table 2). In contrast, the restricted cubic spline analysis indicated a non-linear asso- ciation between total $\mathrm{Ca}$ intake and ischemic stroke ( $P$ for nonlinear trend $=0.006$ ) (Figure 3 ). Both results suggest a threshold effect of $\mathrm{Ca}$ on the risk of ischemic stroke. When comparing the highest quintile to the lowest, dietary $\mathrm{Ca}$ was not linearly associated with the risk of ischemic stroke (Table 2). A borderline non-linear association was also observed for dietary $\mathrm{Ca}$ intake 
Table 2. The HR $(95 \% \mathrm{Cl})$ of ischemic stroke by quintiles of Ca intake in the REGARDS cohort $(n=19,553)$

\begin{tabular}{|c|c|c|c|c|c|c|}
\hline Variable & Quintile 1 & Quintile 2 & Quintile 3 & Quintile 4 & Quintile 5 & $P_{\text {trend }}{ }^{*}$ \\
\hline $\begin{array}{l}\text { Total Ca intake, range } \\
(\mathrm{mg} / \text { day })^{+}\end{array}$ & $77.90-477.6$ & $477.7-703.5$ & 703.6-1,040.9 & $1,041.4-1,559.3$ & $1,559.5-3,822.9$ & \\
\hline No. of cases & 201 & 145 & 154 & 143 & 165 & \\
\hline Person-years & $31,457.10$ & $32,203.45$ & $32,805.39$ & $33,202.72$ & $33,222.93$ & \\
\hline Model 1 & 1.00 & $0.67(0.54-0.83)$ & $0.68(0.55-0.85)$ & $0.65(0.53-0.81)$ & $0.72(0.58-0.89)$ & 0.026 \\
\hline Model 2 & 1.00 & $0.69(0.56-0.86)$ & $0.72(0.58-0.89)$ & $0.70(0.57-0.88)$ & $0.80(0.64-0.99)$ & 0.197 \\
\hline Model 3 & 1.00 & $0.68(0.54-0.84)$ & $0.69(0.54-0.87)$ & $0.67(0.53-0.85)$ & $0.75(0.58-0.98)$ & 0.252 \\
\hline Model 4 & 1.00 & $0.67(0.54-0.84)$ & $0.66(0.52-0.85)$ & $0.65(0.51-0.83)$ & $0.72(0.55-0.95)$ & 0.183 \\
\hline $\begin{array}{l}\text { Dietary Ca intake, range } \\
\text { (mg/day) }\end{array}$ & $76.9-366.1$ & $366.2-512.5$ & $512.6-673.7$ & 673.8-915.6 & $915.7-2,883.7$ & \\
\hline No. of cases & 172 & 159 & 158 & 154 & 165 & \\
\hline Person-years & $31,768.64$ & $32,559.86$ & $32,642.35$ & $32,963.23$ & $32,957.52$ & \\
\hline Model 1 & 1.00 & $0.87(0.70-1.09)$ & $0.85(0.68-1.06)$ & $0.80(0.64-0.99)$ & $0.86(0.69-1.07)$ & 0.200 \\
\hline Model 2 & 1.00 & $0.89(0.72-1.11)$ & $0.87(0.70-1.08)$ & $0.82(0.66-1.03)$ & $0.89(0.72-1.03)$ & 0.356 \\
\hline Model 3 & 1.00 & $0.89(0.71-1.12)$ & $0.87(0.69-1.10)$ & $0.83(0.64-1.06)$ & $0.90(0.67-1.20)$ & 0.568 \\
\hline Model 4 & 1.00 & $0.87(0.70-1.09)$ & $0.82(0.65-1.04)$ & $0.76(0.58-0.99)$ & $0.80(0.59-1.10)$ & 0.193 \\
\hline $\begin{array}{l}\text { Supplemental Ca intake } \\
\text { (mg/day) }\end{array}$ & 0 & $0.1-37.2$ & $92.9-130.0$ & $285.8-1,000.0$ & $1,000.1-1,130.0$ & \\
\hline No. of cases & 327 & 41 & 193 & 106 & 141 & \\
\hline Person-years & $59,968.19$ & $6,923.50$ & $39,074.52$ & $29,498.16$ & $27,427.24$ & \\
\hline Model 1 & 1.00 & $1.20(0.87-1.66)$ & $0.83(0.70-1.00)$ & $0.68(0.55-0.86)$ & $0.90(0.73-1.11)$ & 0.053 \\
\hline Model 2 & 1.00 & $1.19(0.86-1.64)$ & $0.88(0.74-1.06)$ & $0.74(0.59-0.92)$ & $1.00(0.81-1.23)$ & 0.331 \\
\hline Model 3 & 1.00 & $1.20(0.87-1.67)$ & $0.87(0.70-1.07)$ & $0.74(0.59-0.94)$ & $1.01(0.79-1.29)$ & 0.464 \\
\hline Model 4 & 1.00 & $1.22(0.88-1.70)$ & $0.88(0.71-1.10)$ & $0.73(0.58-0.93)$ & $1.03(0.80-1.32)$ & 0.449 \\
\hline
\end{tabular}

Cox proportional hazard regression was used to sequentially adjusted for covariates. Model 1 was adjusted for age, body mass index, sex, race, region, and the interaction of age and race; Model 2 was adjusted for covariates in Model 1, education, income, exercise, smoking, and alcohol; Model 3 was adjusted for variables in Model 2, total energy intake, regular aspirin use, and total magnesium intakes and for the models on dietary $\mathrm{Ca}$ or supplemental $\mathrm{Ca}$, mutually adjusted for dietary, and supplemental Ca; Model 4 was adjusted for variables in Model 3, low density lipoprotein, triglyceride, and vitamin D intake. $\mathrm{HR}$, hazard ratio; $\mathrm{Cl}$, confidence interval; $\mathrm{Ca}$, calcium; REGARDS, Reasons for Geographic And Racial Differences in Stroke.

${ }^{*}$ P for linear trend was calculated by modeling the median of the nutrient for each quintile as a continuous variable; 'Total calcium includes both dietary calcium and supplemental calcium intake.

( $P$ for non-linear trend=0.053) (Supplementary Figure 1). In sensitivity analyses using the quartiles and tertiles of total and dietary $\mathrm{Ca}$, the results remained (data not shown).

In a mediation analysis, the observed association between total $\mathrm{Ca}$ intake and ischemic stroke was mainly mediated through FBG (proportion of association mediated [PAM]: 19.6\%, $P=0.0002)$, type 2 diabetes (PAM: $9 \%, P=0.017$ ), SBP (PAM: $12 \%, P=0.017$ ), hypertension (PAM: $8.0 \%, P=0.014$ ), total cholesterol (PAM: $9.6 \%, P=0.005)$, and HDL (PAM: 9.5\%, $P=0.005$ ) (Supplementary Table 1).

Supplemental $\mathrm{Ca}$ intake was not significantly linearly associated with the risk of ischemic stroke $(\mathrm{HR}, 1.03 ; 95 \% \mathrm{Cl}, 0.80$ to 1.32; $P=0.449$ ) (Table 2). Restricted cubic spline analysis indicated a significant non-linear association between Ca supplement and the risk of ischemic stroke $(P=0.034)$ (Supplementary
Figure 2). Using those without supplemental Ca intake as a reference, the non-linear association remained (data not shown).

In the case-cohort, those in the highest quintile of serum $\mathrm{Ca}$ consumed higher amounts of dietary $\mathrm{Ca}$, had higher serum $\mathrm{Mg}$, and had higher total cholesterol, higher LDL, higher triglycerides, and higher HDL; were more likely to be female and currently drink alcohol as compared to those in the lowest quartile of serum $\mathrm{Ca}(P<0.05)$ (Table 3$)$. An inverse association of serum $\mathrm{Ca}$ levels with ischemic stroke in the case-cohort analysis was observed ( $\mathrm{HR}, 0.73 ; 95 \% \mathrm{Cl}, 0.53$ to $0.99 ; P=0.013$ ) when comparing the highest to the lowest quintile (Table 4). In a sensitivity analysis, further adjusting SBP and type 2 diabetes in the final model, the result remained $(\mathrm{HR}, 0.71 ; 95 \% \mathrm{Cl}, 0.51$ to 0.99; $P=0.016)$. In a sensitivity analysis using data with a reduced sample size ( $n=1,601$, event=602), adjusting for serum 


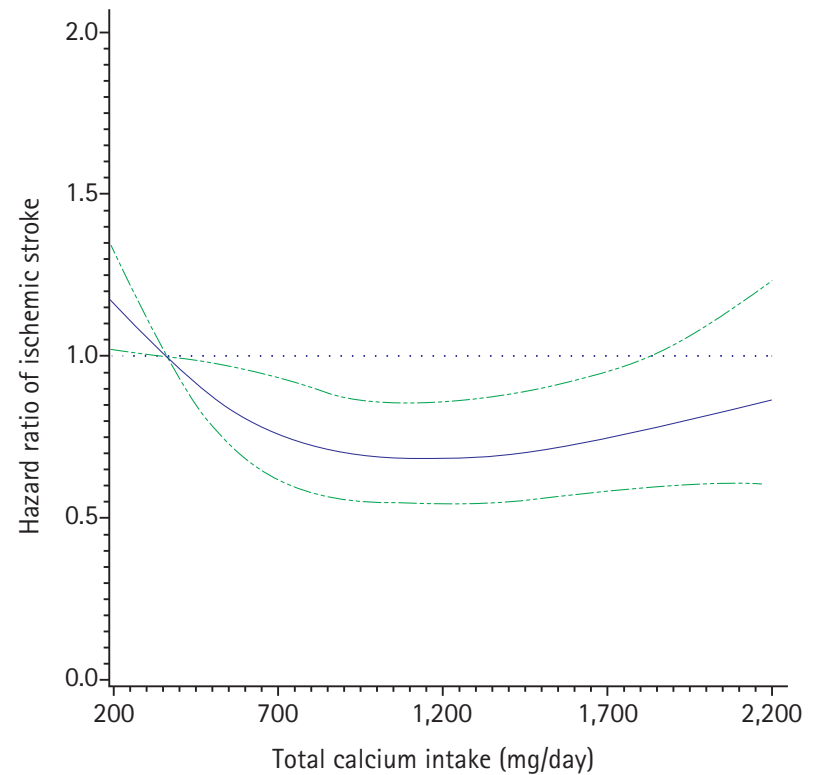

Figure 3. Restricted cubic spline analysis of the association between total calcium (Ca) intake and the risk of ischemic stroke $(n=19,553)$. The middle solid line indicates the point estimates of hazard ratios and the broken lines indicate the lower and upper limits of the corresponding 95\% confidence intervals. The horizontal broken line is at hazard ratio $=1$. Four knots were used for the analysis. In the restricted cubic spline analysis, a significant non-linear association was observed ( $P$ for non-linearity $=0.006$ ).
$25(\mathrm{OH}) \mathrm{D}$, the association disappeared $(\mathrm{HR}, 0.84 ; 95 \% \mathrm{Cl}, 0.57$ to $1.24 ; P=0.568)$. Of note, serum $25(\mathrm{OH}) \mathrm{D}$ was not significant in the model $(P=0.887)$.

The interactions between sex, race or region and $\mathrm{Ca}$ in the models were not statistically significant at alpha $=0.1$.

\section{Discussion}

In this large prospective cohort study, we observed a threshold effect like non-linear inverse association of $\mathrm{Ca}$ intake and an inverse linear association of serum $\mathrm{Ca}$ levels with the risk of ischemic stroke, independent of potential confounding variables. These associations were mainly mediated through diabetes, hypertension, and cholesterol.

Our results are consistent with the findings of two metaanalyses that suggested $\mathrm{Ca}$ intake is non-linearly associated with the risk of stroke. ${ }^{8,9}$ In the Larsson et al. (2013) ${ }^{8}$ metaanalysis, they observed in those with $<700 \mathrm{mg} /$ day mean $\mathrm{Ca}$ intake, the risk of ischemic stroke was lower by $16 \%$ for every $300 \mathrm{mg} /$ day increase in $\mathrm{Ca}$ intake while in those with $>700$ $\mathrm{mg} /$ day mean $\mathrm{Ca}$ intake, the risk of ischemic stroke was higher

Table 3. Baseline characteristics of participants in the case-cohort by quintiles of serum Ca: the REGARDS study (weighted, $n=3,102$ )

\begin{tabular}{|c|c|c|c|c|c|c|}
\hline Characteristic & Quintile 1 & Quintile 2 & Quintile 3 & Quintile 4 & Quintile 5 & $P$ \\
\hline Serum $\mathrm{Ca}$, median $(\mu \mathrm{g} / \mathrm{g})$ & 89.80 & 94.40 & 97.40 & 100.50 & 105.10 & \\
\hline Dietary Ca (mg/day) & $660.36 \pm 364.09$ & $669.10 \pm 336.45$ & $658.08 \pm 343.92$ & $638.32 \pm 331.30$ & $677.49 \pm 346.63$ & 0.013 \\
\hline Supplementary Ca intake (mg/day) & $293.89 \pm 417.23$ & $333.20 \pm 445.90$ & $306.18 \pm 435.20$ & $319.57 \pm 439.66$ & $384.91 \pm 463.65$ & 0.433 \\
\hline Serum $\mathrm{Mg}(\mu \mathrm{g} / \mathrm{g})$ & $18.34 \pm 1.85$ & $19.26 \pm 1.92$ & $19.70 \pm 1.86$ & $19.75 \pm 2.00$ & $19.98 \pm 2.17$ & $<0.0001$ \\
\hline Dietary Mg (mg/day) & $273.63 \pm 122.26$ & $277.46 \pm 116.18$ & $267.62 \pm 113.42$ & $269.60 \pm 121.29$ & $282.30 \pm 125.99$ & 0.365 \\
\hline Supplemental Mg (mg/day) & $43.97 \pm 46.85$ & $45.34 \pm 46.87$ & $41.17 \pm 46.89$ & $45.40 \pm 47.45$ & $49.24 \pm 47.02$ & 0.188 \\
\hline Age (yr) & $66.02 \pm 10.12$ & $66.96 \pm 9.91$ & $66.31 \pm 9.26$ & $65.53 \pm 8.48$ & $65.59 \pm 9.17$ & 0.094 \\
\hline BMI $\left(\mathrm{kg} / \mathrm{m}^{2}\right)$ & $29.85 \pm 6.07$ & $28.81 \pm 6.00$ & $28.80 \pm 5.90$ & $29.10 \pm 38.93$ & $28.96 \pm 5.67$ & 0.020 \\
\hline Fasting blood glucose (mg/dL) & $105.66 \pm 37.77$ & $103.14 \pm 30.80$ & $105.05 \pm 40.13$ & $106.68 \pm 38.93$ & $104.82 \pm 36.17$ & 0.709 \\
\hline $\mathrm{DBP}(\mathrm{mm} \mathrm{Hg})$ & $76.18 \pm 10.17$ & $76.88 \pm 9.38$ & $76.27 \pm 9.53$ & $76.87 \pm 9.36$ & $76.79 \pm 9.91$ & 0.643 \\
\hline $\mathrm{SBP}(\mathrm{mm} \mathrm{Hg})$ & $129.13 \pm 18.29$ & $128.96 \pm 17.31$ & $128.59 \pm 16.17$ & $128.40 \pm 16.47$ & $128.85 \pm 17.74$ & 0.986 \\
\hline Total cholesterol (mg/dL) & $184.38 \pm 36.40$ & $186.31 \pm 38.86$ & $190.95 \pm 39.65$ & $193.37 \pm 38.86$ & $201.59 \pm 42.63$ & $<0.0001$ \\
\hline $\mathrm{HDL}(\mathrm{mg} / \mathrm{dL})$ & $49.11 \pm 15.09$ & $51.16 \pm 15.40$ & $50.62 \pm 15.80$ & $52.33 \pm 16.42$ & $53.79 \pm 17.89$ & $<0.0001$ \\
\hline LDL (mg/dL) & $109.62 \pm 32.74$ & $109.60 \pm 34.80$ & $113.78 \pm 33.52$ & $114.93 \pm 33.27$ & $119.66 \pm 36.38$ & $<0.0001$ \\
\hline Triglyceride (mg/dL) & $130.47 \pm 82.91$ & $128.29 \pm 77.47$ & $134.11 \pm 106.29$ & $131.71 \pm 77.07$ & $141.69 \pm 91.07$ & 0.010 \\
\hline Calories (kcal/day) & $1,712.07 \pm 729.66$ & $1,741.06 \pm 728.13$ & $1,654.51 \pm 681.61$ & $1,732.97 \pm 739.52$ & $1,716.33 \pm 707.74$ & 0.539 \\
\hline Female sex $(\%)$ & 48.63 & 51.68 & 49.35 & 53.34 & 61.92 & $<0.0001$ \\
\hline Black race $(\%)$ & 38.13 & 39.04 & 39.35 & 41.44 & 42.56 & 0.483 \\
\hline Regular aspirin use (\%) & 46.04 & 42.88 & 45.97 & 46.98 & 46.56 & 0.619 \\
\hline Current alcohol use (\%) & 44.26 & 54.24 & 53.55 & 47.47 & 53.44 & 0.003 \\
\hline
\end{tabular}

Values are presented as mean \pm standard deviation. Analysis of variance and Kruskal-Wallis test were used to compare continuous variables across serum Ca quintiles. Chi-square test was used to test the association of categorical variables with quintiles of serum Ca.

Ca, calcium; REGARDS, Reasons for Geographic And Racial Differences in Stroke; Mg, magnesium; BMI, body mass index; DBP, diastolic blood pressure; SBP, systolic blood pressure; HDL, high density lipoprotein; LDL, low density lipoprotein. 
by $3 \%$. In the same study, the authors stated that Ca has a beneficial effect in Asian populations with low to moderate $\mathrm{Ca}$ intake but not in American or European populations with high $\mathrm{Ca}$ intakes. The studies with mean Ca intake $>700 \mathrm{mg} /$ day were mostly European with only one American study. None of the individual studies in the Larsson meta-analysis with mean $\mathrm{Ca}$ intake $>700 \mathrm{mg} /$ day found a significant association. Of note, the sample sizes in all the studies included in the Larsson et al. ${ }^{8}$ meta-analysis were small. Unlike previous studies that suggested the possible benefit of dietary $\mathrm{Ca}$ in reducing the risk of stroke is observed only among the Asian populations with low to moderate $\mathrm{Ca}$ intake, we observed inverse or non-linear inverse associations between $\mathrm{Ca}$ and ischemic stroke in Americans. In the Tian et al. (2015) ${ }^{9}$ updated meta-analysis, the authors reported an inverse association in the studies with a long duration of follow-up ( $\geq 14$ years) and in the studies with dairy Ca sources. They did not find a significant association in short duration studies and studies with non-dairy sources of $\mathrm{Ca}$, but the overall pooled result showed a significant inverse association. The difference between our study and the other studies in the meta-analysis might be due to differences in study population (in our case, participants from stroke belt region and Black participants were oversampled); the other potential reasons might be due to differences in adjusted variables. As hypertension and type 2 diabetes were on the path of association, we did not adjust them. The Tian et al. ${ }^{9}$ study hypothesized that the role of $\mathrm{Ca}$ in stroke might be due to the beneficial effect of Ca on hypertension. In our study, we confirmed that hypertension and type 2 diabetes were two possible mediators of the association between $\mathrm{Ca}$ and stroke. Notably, none of the previous studies found a non-linear association, presumably limited by the statistical power.

The discrepancy in the association of total $\mathrm{Ca}$ and dietary $\mathrm{Ca}$ intake with ischemic stroke (a threshold effect like non-linear association of total $\mathrm{Ca}$ and less clear pattern and borderline non-linear association for dietary $\mathrm{Ca}$ ) may be explained by multiple factors, including sources of $\mathrm{Ca}$ intake and bioavailability. The absorption of $\mathrm{Ca}$ taken from food is affected by the source of the food, which may have Ca bound in oxalate or phytate, while Ca from supplements may be more readily absorbed..$^{37}$ Generally, about 30\% of Ca from food is absorbed but the bioavailability varies by the source of $\mathrm{Ca}^{38}$ Mean (fractional) $\mathrm{Ca}$ absorption is directly proportional with intake at high intake. While $\mathrm{Ca}$ is mostly actively transported from the intestinal lumen, at high levels passive diffusion is involved in $\mathrm{Ca}$ absorption. ${ }^{39}$ Individuals that take Ca supplements may have higher socioeconomic status and may be more health conscious as well. ${ }^{39,40}$

Though participants with high $\mathrm{Ca}$ intake also tend to have high serum $\mathrm{Ca}$, serum $\mathrm{Ca}$ is tightly regulated by parathyroid hormone and calcitonin. Parathyroid hormone maintains the level of serum $\mathrm{Ca}$ by increasing bone resorption, renal reabsorption, and intestinal absorption by activating vitamin $\mathrm{D}$ in the kidney. ${ }^{41}$ In contrast, calcitonin reduces serum $\mathrm{Ca}$ by prevention of bone resorption. ${ }^{42}$ Thus, the level of serum $\mathrm{Ca}$ is hormonally regulated by parathyroid hormone, vitamin $\mathrm{D}$ and calcitonin and is not only affected by the amount of $\mathrm{Ca}$ intake. In the present study, we have adjusted for vitamin $D$ intake in the cohort analysis and in a sensitivity analysis for serum $25(\mathrm{OH}) \mathrm{D}$ in the case-cohort analysis. After adjusting for vitamin $D$, the association for total $\mathrm{Ca}$ became stronger but slightly attenuated for serum $\mathrm{Ca}$. We did not have data to adjust for sex hormones but experimental studies indicate sex hormones impact cardiac contractility and Ca homeostasis. ${ }^{43}$

The pathophysiology of ischemic stroke involves atherosclerosis that narrows arterial blood vessels supplying blood to the

Table 4. The HR (95\% Cl) of ischemic stroke by quintiles of serum Ca in the case-cohort analysis (weighted, $n=3,102)$

\begin{tabular}{|c|c|c|c|c|c|c|}
\hline Variable & Quintile 1 & Quintile 2 & Quintile 3 & Quintile 4 & Quintile 5 & $P_{\text {trend }}{ }^{*}$ \\
\hline Serum $C a$, range $(\mu \mathrm{g} / \mathrm{g})$ & $67.6-92.5$ & $92.6-95.9$ & $96.0-98.80$ & $98.9-102.3$ & $102.4-150.5$ & \\
\hline No. of cases & 161 & 160 & 151 & 129 & 129 & \\
\hline Person-years & $4,345.59$ & $4,232.65$ & $4,389.08$ & $4,461.59$ & $4,463.73$ & \\
\hline Model 1 & 1.00 & $0.97(0.72-1.30)$ & $0.92(0.69-1.24)$ & $0.80(0.59-1.08)$ & $0.77(0.57-1.05)$ & 0.046 \\
\hline Model 2 & 1.00 & $0.99(0.74-1.34)$ & $0.95(0.71-1.27)$ & $0.78(0.57-1.06)$ & $0.74(0.54-1.00)$ & 0.017 \\
\hline Model 3 & 1.00 & $1.01(0.75-1.37)$ & $0.97(0.71-1.30)$ & $0.79(0.57-1.09)$ & $0.74(0.54-1.02)$ & 0.026 \\
\hline Model 4 & 1.00 & $1.01(0.76-1.35)$ & $0.95(0.71-1.26)$ & $0.79(0.58-1.07)$ & $0.73(0.53-0.99)$ & 0.013 \\
\hline
\end{tabular}

Weighted Cox proportional hazard regression with robust sandwich estimate for the variance was used to fit the models. Model 1 was adjusted for age, sex, race, and the interaction of age and race; Model 2 was adjusted for covariates in Model 1, education, income, smoking, and alcohol; Model 3 was adjusted for variables in Model 2, regular aspirin use, and dietary Ca intake; Model 4 was adjusted for variables in Model 3, low density lipoprotein, triglyceride, and serum magnesium.

$\mathrm{HR}$, hazard ratio; $\mathrm{Cl}$, confidence interval; $\mathrm{Ca}$, calcium.

${ }^{*}$ P for linear trend was calculated by modeling the median of serum $\mathrm{Ca}$ for each quintile as a continuous variable. 
brain or occlusion of the blood vessels with thromboembolus originating in the heart or other blood vessels resulting in reduced blood flow to the neurons. ${ }^{44}$ The mechanism through which $\mathrm{Ca}$ is related to lower risk of ischemic stroke may include reducing platelet aggregation, ${ }^{45}$ blood cholesterol, ${ }^{46,47}$ blood pressure, ${ }^{1,2}$ and the risk of type 2 diabetes. ${ }^{48,49}$ In this study, type 2 diabetes, FBG, SBP, hypertension, HDL, and total cholesterol significantly mediated the associations between $\mathrm{Ca}$ intake and ischemic stroke. The mediation by cholesterol was evident after adjusting for vitamin $D$ intake. Previous studies found $\mathrm{Ca}$ supplementation reduced insulin resistance. ${ }^{50,51}$ The nature of the mediation may be more complex than described in this study; for example, the effect of type 2 diabetes may be through worsening hypertension given that type 2 diabetes affects the vascular system. While increases in intracellular $\mathrm{Ca}$ have been implicated in ischemic injury in ischemic stroke, higher serum $\mathrm{Ca}$ has been reported to be associated with reduced ischemic tissue injury in ischemic stroke by affecting excitotoxic pathways and ischemic preconditioning. ${ }^{52}$ The nonlinear association between $\mathrm{Ca}$ intake and the risk of ischemic stroke might be explained by the inadequacy of the protective physiologic benefits at low intake and the dominance of pathologic effects such as cerebrovascular calcifications and atherosclerosis at high intake..$^{53}$ In addition, the pattern and source of $\mathrm{Ca}$ intake might be different at low and high intakes. Those with inadequate $\mathrm{Ca}$ intake might be consuming $\mathrm{Ca}$ through dietary sources low in $\mathrm{Ca}$ while those with high total intake might be taking high levels of $\mathrm{Ca}$ supplements, which might increase the risk of arterial calcification. There is evidence of increased cardiovascular events with increased supplemental $\mathrm{Ca}$ intake, but not with increased dietary Ca intake. ${ }^{12}$

There are some limitations relevant to the interpretation of the results of this study. First, because of the small number of hemorrhagic strokes, we could not conduct subtype analysis. Second, the study was restricted to only blacks and non-Hispanic whites, which may limit the generalizability of the results to other races. Third, we cannot rule out residual confounding, for example, by type of supplement, and sex hormones given the study is an observational study. Fourth, Ca intake and serum Ca were measured only once, and we could not account for change in dietary habits over the follow-up period. Finally, for the case-cohort analysis, serum Ca was not corrected for albumin-bound $\mathrm{Ca}_{1}$ and for the cohort analysis, bioavailability for $\mathrm{Ca}$ intake that varies by the food sources, supplement type, and participant characteristics was not accounted for. This study also has several strengths. The prospective cohort design reduced recall bias and the long duration of follow-up with a large number of cases of ischemic stroke conferred enough power to detect clinically meaningful differences. It is unique in that we tested the non-linear association of $\mathrm{Ca}$ intake with the risk of ischemic stroke. Previous meta-analyses have suggested that $\mathrm{Ca}$ was non-linearly associated with the risk of ischemic, but to the best of our knowledge, this is the first original prospective cohort study to examine non-linear associations between $\mathrm{Ca}$ intake and the risk of ischemic stroke. We also conducted case-cohort analysis on the association of serum $\mathrm{Ca}$ with the risk of ischemic stroke to corroborate the findings from the nutrient intake with generally consistent results, which further strengthened our study. Furthermore, we confirmed the mediation of the association between total $\mathrm{Ca}$ intake and ischemic stroke by type 2 diabetes and hypertension.

\section{Conclusions}

In conclusion, Ca intake has a threshold effect like non-linear association with risk of ischemic stroke. Type 2 diabetes and hypertension may mediate the association. Given the growing concern on the role of $\mathrm{Ca}$ intake in cardiovascular and cerebrovascular health and the variable results from individual studies, larger prospective cohort studies with repeated measurements of $\mathrm{Ca}$ intake, and randomized controlled trials are needed to elucidate the beneficial and detrimental role of $\mathrm{Ca}$ intake.

\section{Supplementary materials}

Supplementary materials related to this article can be found online at https://doi.org/10.5853/jos.2019.00542.

\section{Disclosure}

The authors have no financial conflicts of interest.

\section{Acknowledgments}

This work was supported by a research grant from the NIH (R01ES021735). REGARDS is supported by a cooperative agreement U01 NS041588 from the National Institute of Neurological Disorders and Stroke, National Institutes of Health, Department of Health and Human Service. The content is solely the responsibility of the authors and does not necessarily represent the official views of the National Institute of Neurological Disorders and Stroke or the National Institutes of Health. Representatives of the funding agency have been involved in the review of the manuscript but not directly involved in the collection, management, analysis or interpretation of the data. The authors thank the other investigators, the staff, and the partic- 
ipants of the REGARDS study for their valuable contributions. A full list of participating REGARDS investigators and institutions can be found at http://www.regardsstudy.org.

\section{References}

1. van Mierlo LA, Arends LR, Streppel MT, Zeegers MP, Kok FJ, Grobbee DE, et al. Blood pressure response to calcium supplementation: a meta-analysis of randomized controlled trials. J Hum Hypertens 2006;20:571-580.

2. Bucher HC, Cook RJ, Guyatt GH, Lang JD, Cook DJ, Hatala R, et al. Effects of dietary calcium supplementation on blood pressure. A meta-analysis of randomized controlled trials. JAMA 1996;275:1016-1022.

3. Kass L, Weekes J, Carpenter L. Effect of magnesium supplementation on blood pressure: a meta-analysis. Eur J Clin Nutr 2012;66:411-418.

4. Jee SH, Miller ER 3rd, Guallar E, Singh VK, Appel $\amalg$, Klag MJ. The effect of magnesium supplementation on blood pressure: a meta-analysis of randomized clinical trials. $\mathrm{Am} \mathrm{J} \mathrm{Hy-}$ pertens 2002;15:691-696.

5. Schulze MB, Schulz M, Heidemann C, Schienkiewitz A, Hoffmann $\mathrm{K}$, Boeing $\mathrm{H}$. Fiber and magnesium intake and incidence of type 2 diabetes: a prospective study and metaanalysis. Arch Intern Med 2007;167:956-965.

6. Guerrero-Romero F, Tamez-Perez HE, González-González G, Salinas-Martínez AM, Montes-Villarreal J, Treviño-Ortiz JH, et al. Oral magnesium supplementation improves insulin sensitivity in non-diabetic subjects with insulin resistance. A double-blind placebo-controlled randomized trial. Diabetes Metab 2004;30:253-258.

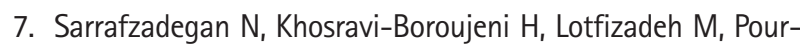
mogaddas A, Salehi-Abargouei A. Magnesium status and the metabolic syndrome: a systematic review and meta-analysis. Nutrition 2016;32:409-417.

8. Larsson SC, Orsini N, Wolk A. Dietary calcium intake and risk of stroke: a dose-response meta-analysis. Am J Clin Nutr 2013; 97:951-957.

9. Tian DY, Tian J, Shi CH, Song B, Wu J, Ji Y, et al. Calcium intake and the risk of stroke: an up-dated meta-analysis of prospective studies. Asia Pac J Clin Nutr 2015;24:245-252.

10. Rohrmann $\mathrm{S}$, Garmo H, Malmström H, Hammar N, Jungner I, Walldius $\mathrm{G}$, et al. Association between serum calcium concentration and risk of incident and fatal cardiovascular disease in the prospective AMORIS study. Atherosclerosis 2016;251:85-93.

11. Chowdhury R, Stevens S, Ward H, Chowdhury S, Sajjad A, Franco $\mathrm{OH}$. Circulating vitamin $\mathrm{D}$, calcium and risk of cere- brovascular disease: a systematic review and meta-analysis. Eur J Epidemiol 2012;27:581-591.

12. Anderson JJ, Kruszka B, Delaney JA, He K, Burke GL, Alonso A, et al. Calcium intake from diet and supplements and the risk of coronary artery calcification and its progression among older adults: 10-year follow-up of the Multi-Ethnic Study of Atherosclerosis (MESA). J Am Heart Assoc 2016;5:e003815.

13. Bolland MJ, Grey A, Reid IR. Calcium supplements and cardiovascular risk: 5 years on. Ther Adv Drug Saf 2013;4:199210.

14. Wang L, Manson JE, Sesso HD. Calcium intake and risk of cardiovascular disease: a review of prospective studies and randomized clinical trials. Am J Cardiovasc Drugs 2012;12: 105-116.

15. Bolland MJ, Avenell A, Baron JA, Grey A, MacLennan GS, Gamble GD, et al. Effect of calcium supplements on risk of myocardial infarction and cardiovascular events: meta-analysis. BMJ 2010;341:c3691.

16. Howard VJ, Kleindorfer DO, Judd SE, McClure LA, Safford $M M$, Rhodes JD, et al. Disparities in stroke incidence contributing to disparities in stroke mortality. Ann Neurol 2011;69: 619-627.

17. Judd SE, Kleindorfer DO, McClure LA, Rhodes JD, Howard G, Cushman $\mathrm{M}$, et al. Self-report of stroke, transient ischemic attack, or stroke symptoms and risk of future stroke in the REasons for Geographic And Racial Differences in Stroke (REGARDS) study. Stroke 2013;44:55-60.

18. Howard VJ, Cushman M, Pulley L, Gomez CR, Go RC, Prineas $\mathrm{RJ}$, et al. The reasons for geographic and racial differences in stroke study: objectives and design. Neuroepidemiology 2005;25:135-143.

19. Jensen-Otsu $E_{1}$ Austin GL. Antidepressant use is associated with increased energy intake and similar levels of physical activity. Nutrients 2015;7:9662-9671.

20. Block G, Hartman AM, Dresser CM, Carroll MD, Gannon J, Gardner L. A data-based approach to diet questionnaire design and testing. Am J Epidemiol 1986;124:453-469.

21. Aaron KJ, Campbell RC, Judd SE, Sanders PW, Muntner P. Association of dietary sodium and potassium intakes with albuminuria in normal-weight, overweight, and obese participants in the Reasons for Geographic and Racial Differences in Stroke (REGARDS) Study. Am J Clin Nutr 2011;94:10711078.

22. Newby PK, Noel SE, Grant R, Judd S, Shikany JM, Ard J. Race and region have independent and synergistic effects on dietary intakes in black and white women. Nutr J 2012;11:25.

23. Boucher B, Cotterchio M, Kreiger N, Nadalin V, Block T, Block G. Validity and reliability of the Block98 food-frequency 
questionnaire in a sample of Canadian women. Public Health Nutr 2006:9:84-93.

24. Subar AF, Thompson FE, Kipnis V, Midthune D, Hurwitz P, McNutt $\mathrm{S}$, et al. Comparative validation of the Block, Willett, and National Cancer Institute food frequency questionnaires: the Eating at America's Table Study. Am J Epidemiol 2001;154: 1089-1099.

25. Tsivgoulis G, Psaltopoulou T, Wadley VG, Alexandrov AV, Howard G, Unverzagt FW, et al. Adherence to a Mediterranean diet and prediction of incident stroke. Stroke 2015;46: 780-785.

26. Judd SE, Gutiérrez OM, Newby PK, Howard G, Howard VJ, Locher $\mathrm{JL}$, et al. Dietary patterns are associated with incident stroke and contribute to excess risk of stroke in black Americans. Stroke 2013;44:3305-3311.

27. Stroke: 1989. Recommendations on stroke prevention, diagnosis, and therapy. Report of the WHO task force on stroke and other cerebrovascular disorders. Stroke 1989;20:1407-1431.

28. O'Neal WT, Qureshi WT, Judd SE, Meschia JF, Howard VJ, Howard $G$, et al. Heart rate and ischemic stroke: the REasons for Geographic And Racial Differences in Stroke (REGARDS) study. Int J Stroke 2015;10:1229-1235.

29. Shrira I, Christenfeld N, Howard G. Exposure to the US Stroke Buckle as a risk factor for cerebrovascular mortality. Neuroepidemiology 2008;30:229-233.

30. Hardwick LL, Jones MR, Brautbar N, Lee DB. Magnesium absorption: mechanisms and the influence of vitamin $\mathrm{D}$, calcium and phosphate. J Nutr 1991;121:13-23.

31. Tao MH, Dai $Q$, Millen AE, Nie J, Edge SB, Trevisan M, et al. Associations of intakes of magnesium and calcium and survival among women with breast cancer: results from Western New York Exposures and Breast Cancer (WEB) Study. Am J Cancer Res 2015;6:105-113.

32. Li R, Hertzmark E, Louie M, Chen L, Spiegelman D. The SAS LGTPHCURV9 Macro. https://cdn1.sph.harvard.edu/wp-content/uploads/sites/271/2012/09/lgtphcurv9_7-3-2011.pdf. 2011. Accessed August 30, 2019.

33. Hertzmark E, Pazaris M, Spiegelman D. The SAS MEDIATE Macro. https://cdn1.sph.harvard.edu/wp-content/uploads/ sites/271/2012/09/mediate_manual_2012_06_06.pdf. 2012. Accessed August 30, 2019.

34. VanderWeele TJ. Mediation analysis: a practitioner's guide. Annu Rev Public Health 2016;37:17-32.

35. Kulathinal S, Karvanen J, Saarela O, Kuulasmaa K. Case-cohort design in practice: experiences from the MORGAM Project. Epidemiol Perspect Innov 2007;4:15.

36. Barlow WE, Ichikawa L, Rosner D, Izumi S. Analysis of casecohort designs. J Clin Epidemiol 1999;52:1165-1172.
37. Amalraj A, Pius A. Bioavailability of calcium and its absorption inhibitors in raw and cooked green leafy vegetables commonly consumed in India: an in vitro study. Food Chem 2015;170:430-436.

38. Overview of calcium. In: Ross AC, Taylor $C L$, Yaktine AL, Del Valle HB; Committee to Review Dietary Reference Intakes for Vitamin D and Calcium. Dietary Reference Intakes for Calcium and Vitamin D. Washington, DC: National Academies Press (US), 2011:35-74. https://www.ncbi.nlm.nih.gov/books/ NBK56060/.

39. Vatanparast $H$, Adolphe JL, Whiting SJ. Socio-economic status and vitamin/mineral supplement use in Canada. Health Rep 2010;21:19-25.

40. Sebastian RS, Cleveland LE, Goldman JD, Moshfegh AJ. Older adults who use vitamin/mineral supplements differ from nonusers in nutrient intake adequacy and dietary attitudes. J Am Diet Assoc 2007;107:1322-1332.

41. Goltzman D, Mannstadt M, Marcocci C. Physiology of the calcium-parathyroid hormone-vitamin D axis. Front Horm Res 2018;50:1-13.

42. Ikegame $M$, Ejiri $S$, Ozawa $H$. Calcitonin-induced change in serum calcium levels and its relationship to osteoclast morphology and number of calcitonin receptors. Bone 2004;35: 27-33.

43. Ayaz 0, Howlett SE. Testosterone modulates cardiac contraction and calcium homeostasis: cellular and molecular mechanisms. Biol Sex Differ 2015;6:9.

44. Deb P, Sharma S, Hassan KM. Pathophysiologic mechanisms of acute ischemic stroke: an overview with emphasis on therapeutic significance beyond thrombolysis. Pathophysiology 2010;17:197-218.

45. Saito K, Sano H, Kawahara J, Yokoyama M. Calcium supplementation attenuates an enhanced platelet function in saltloaded mildly hypertensive patients. Hypertension 1995;26: 156-163.

46. Bell L, Halstenson CE, Halstenson CJ, Macres M, Keane WF. Cholesterol-lowering effects of calcium carbonate in patients with mild to moderate hypercholesterolemia. Arch Intern Med 1992;152:2441-2444.

47. Chrysant SG, Chrysant GS. Controversy regarding the association of high calcium intake and increased risk for cardiovascular disease. J Clin Hypertens (Greenwich) 2014;16:545-550.

48. Liu S, Song Y, Ford ES, Manson JE, Buring JE, Ridker PM. Dietary calcium, vitamin $\mathrm{D}$, and the prevalence of metabolic syndrome in middle-aged and older U.S. women. Diabetes Care 2005;28:2926-2932.

49. Pittas AG, Lau J, Hu FB, Dawson-Hughes B. The role of vitamin $\mathrm{D}$ and calcium in type 2 diabetes. A systematic review and 
meta-analysis. J Clin Endocrinol Metab 2007;92:2017-2029.

50. Sánchez $M$, de la Sierra A, Coca A, Poch E, Giner V, UrbanoMárquez A. Oral calcium supplementation reduces intraplatelet free calcium concentration and insulin resistance in essential hypertensive patients. Hypertension 1997;29:531-536.

51. Pikilidou MI, Lasaridis AN, Sarafidis PA, Befani CD, Koliakos GG, Tziolas IM, et al. Insulin sensitivity increase after calcium supplementation and change in intraplatelet calcium and sodium-hydrogen exchange in hypertensive patients with type 2 diabetes. Diabet Med 2009;26:211-219.

52. Buck BH, Liebeskind DS, Saver JL, Bang OY, Starkman S, Ali LK, et al. Association of higher serum calcium levels with smaller infarct volumes in acute ischemic stroke. Arch Neurol 2007;64:1287-1291.

53. Koton S, Tashlykov V, Schwammenthal Y, Molshatzki N, Merzeliak 0 , Tsabari $R$, et al. Cerebral artery calcification in patients with acute cerebrovascular diseases: determinants and long-term clinical outcome. Eur J Neurol 2012;19:739-745. 
Supplementary Table 1. The proportion (\%) of the association between total calcium intake and ischemic stroke mediated by covariates

\begin{tabular}{lcc}
\hline Variable & Proportion mediated (95\% Cl) & $P$ \\
\hline Glucose & $19.6(4.0-58.9)$ & $<.001$ \\
Type 2 diabetes & $9.0(1.7-36.0)$ & 0.017 \\
SBP & $12.0(2.4-43.6)$ & 0.006 \\
Hypertension & $8.0(1.5-32.8)$ & 0.014 \\
Total cholesterol & $9.6(2.2-33.4)$ & 0.005 \\
High total cholesterol & $2.1(0.3-13.4)$ & 0.089 \\
HDL & $9.5(2.2-33.2)$ & 0.005 \\
\hline
\end{tabular}

The models were adjusted for age, body mass index, sex, income, education, region, alcohol intake, physical activity, smoking, low density lipoprotein, triglyceride, and total energy intake.

$\mathrm{Cl}$, confidence interval; SBP, systolic blood pressure; HDL, high density lipoprotein. 


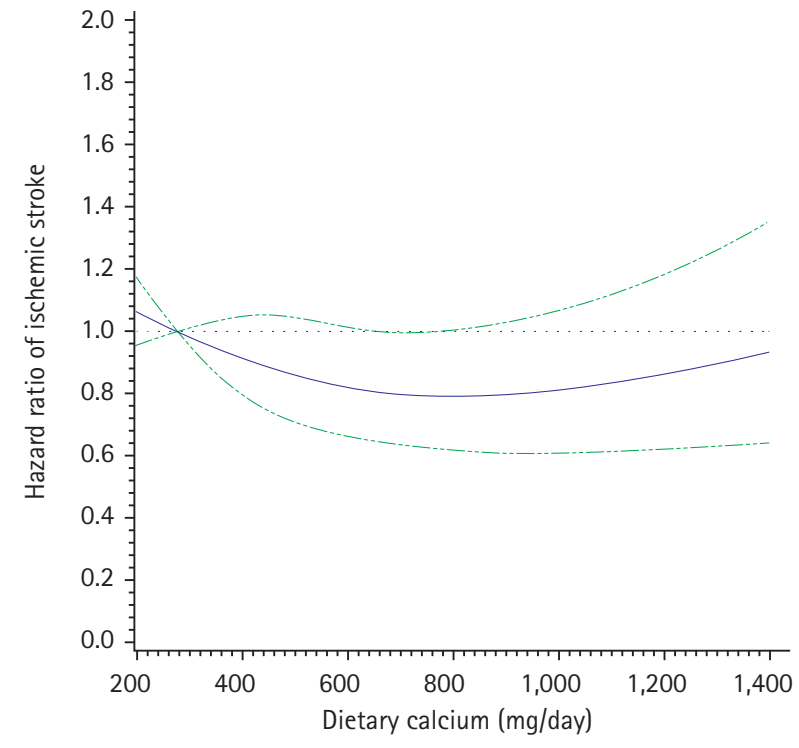

Supplementary Figure 1. Restricted cubic spline of dietary calcium (Ca) intake and the hazard ratios of ischemic stroke $(n=19,553)$. The middle solid line indicates the point estimates and the broken lines indicate the lower and upper limits of the corresponding 95\% confidence intervals. The horizontal broken line is at hazard ratio=1. Four knots were used for the analysis. In the restricted cubic spline analysis, a borderline non-linear association was observed ( $P$ for non-linearity=0.053).

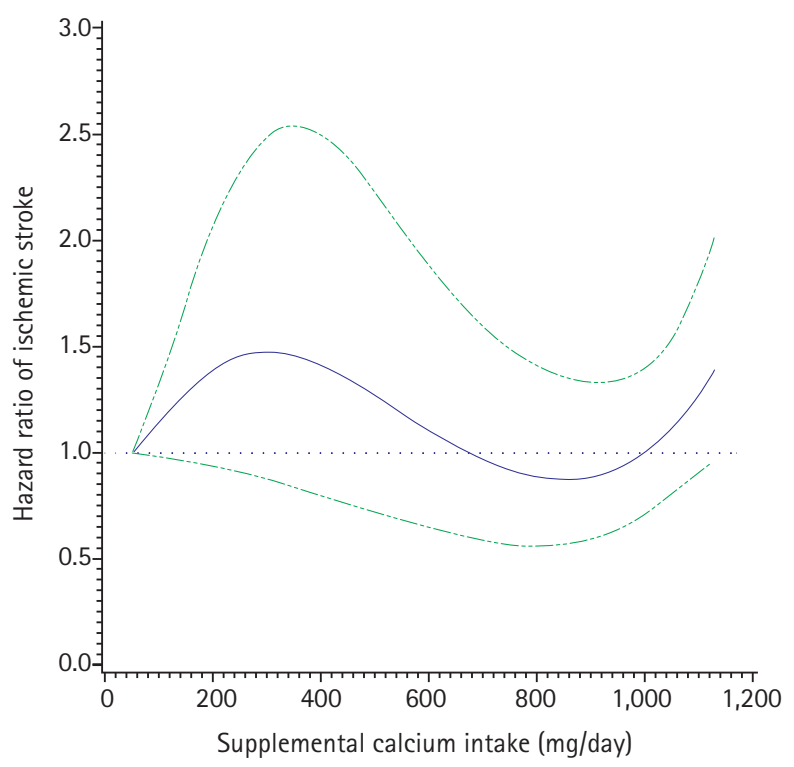

Supplementary Figure 2. Restricted cubic spline of supplemental calcium (Ca) intake and the hazard ratios of ischemic stroke $(n=19,553)$. The middle solid line indicates the point estimates and the broken lines indicate the lower and upper limits of the corresponding 95\% confidence intervals. The horizontal broken line is at hazard ratio $=1$. Four knots were used for the analysis. In the restricted cubic spline analysis, a significant non-linear association was observed ( $P$ for non-linearity=0.034) 